\title{
Facilitation of Stepping with Epidural Stimulation in Spinal Rats: Role of Sensory Input
}

\author{
Igor Lavrov, ${ }^{1}$ Grégoire Courtine, ${ }^{4}$ Christine J. Dy, ${ }^{1}$ Rubia van den Brand, ${ }^{4}$ Andy J. Fong, ${ }^{5}$ Yuri Gerasimenko, ${ }^{1,6}$ \\ Hui Zhong, ${ }^{1}$ Roland R. Roy, ${ }^{1,3}$ and V. Reggie Edgerton ${ }^{1,2,3}$ \\ Departments of ${ }^{1}$ Physiological Science and ${ }^{2}$ Neurobiology, and ${ }^{3}$ Brain Research Institute, University of California, Los Angeles, Los Angeles, California \\ 90095-1527, ${ }^{4}$ University of Zurich, CH-8006 Zurich, Switzerland, ${ }^{5}$ Bioenginering Option, California Institute of Technology, Pasadena, California 91125 , and \\ ${ }^{6}$ Pavlov Institute of Physiology, St. Petersburg 199034, Russia
}

We investigated the role of afferent information during recovery of coordinated rhythmic activity of the hindlimbs in rats with a complete spinal cord section (approximately T8) and unilateral deafferentation (T12-S2) to answer the following questions: (1) Can bilateral stepping be generated with only afferent projections intact on one side? (2) Can the sensory input from the non-deafferented side compensate for the loss of the afferent input from the deafferented side through the crossed connections within the lumbosacral spinal cord? (3) Which afferent projections to the spinal cord from the non-deafferented side predominantly mediate the effect of epidural stimulation to facilitate stepping? Recovery of stepping ability was tested under the facilitating influence of epidural stimulation at the S1 spinal segment, or epidural stimulation plus quipazine, a 5-HT agonist. All chronic spinal rats were able to generate stepping-like patterns on a moving treadmill on the non-deafferented, but not deafferented, side from 3 to 7 weeks after surgery when facilitated by epidural stimulation. Adaptation to the loss of unilateral afferent input was evident at 7 weeks after surgery, when some movements occurred on the deafferented side. Spinal-cord-evoked potentials were observed on both sides, although middle (monosynaptic) and late (long latency) responses were more prominent on the non-deafferented side. The afferent information arising from the non-deafferented side, however, eventually could mediate limited restoration of hindlimb movements on the deafferented side. These data suggest that facilitation of stepping with epidural stimulation is mediated primarily through ipsilateral afferents that project to the locomotor networks.

Key words: spinal cord; spinal cord injury; deafferentation; synaptic plasticity; spinal cord stimulation; CPG

\section{Introduction}

Goldberger (1988) reported excellent recovery of motor function after deafferentation with a spared root compared with a more impaired locomotor pattern after a complete unilateral deafferentation in cats. This observation emphasized the importance of afferent feedback in the control of locomotion but also showed that the loss of afferent information can be compensated for by an increased contribution of descending control. Based on evidence indicating a critical role for afferent information in the recovery of coordinated stepping in chronic spinal animals, a number of motor training strategies have been developed to maximize the use of afferent input in promoting recovery of locomotor capacities after a spinal cord injury (Edgerton et al., 1991, 2001; Rossignol, 2006).

Another strategy to facilitate motor performance after a spinal cord injury is the modulation of afferent input via epi-

\footnotetext{
Received March 11, 2008; revised May 17, 2008; accepted June 9, 2008.

This work was supported by National Institutes of Health Grant NS16333, Russian Foundation for Basic Research (RFBR)-U.S. Civilian Research and Development Foundation 07-04-91106, RFBR 07-04-00526, and the Christopher Reeve Paralysis Foundation (Spinal Cord Consortium, Springfield, NJ). We thank Sharon Zdunowski for technical assistance and constructive comments, and Maynor Herrera for excellent animal care.

The authors declare no competing financial interests.

Correspondence should be addressed to Dr. V. Reggie Edgerton, Department of Physiological Science, University of California, Los Angeles, 621 Charles E. Young Drive LS 1804, Los Angeles, CA 90095-1527. E-mail: vre@ucla.edu. DOI:10.1523/JNEUROSCI.1069-08.2008

Copyright $\odot 2008$ Society for Neuroscience $\quad$ 0270-6474/08/287774-07\$15.00/0
}

dural stimulation (ES). ES has been applied successfully as a method to enhance stepping in animals (Gerasimenko et al., 2003, 2006, 2007; Ichiyama et al., 2005) and in humans (Dimitrijevic et al., 1998; Dimitrijevic and Dimitrijevic, 2002; Herman et al., 2002) after spinal cord injury and has been associated with modulation of afferent input at the dorsal root level (Coburn et al., 1985; Holsheimer, 1998). Recently, we have used ES to investigate the spinal cord circuits that are involved in the modulation of stepping patterns in intact (Gerasimenko et al., 2006) and complete (Lavrov et al., 2006a) spinal rats. We observed modulation of spinal cord motor-evoked potentials in a phase-dependent manner during bipedal stepping in intact rats (Gerasimenko et al., 2006) and in nondisabled humans (Courtine et al., 2007). We also found that the reappearance of polysynaptic components of the evoked potentials after a complete injury generally coincides with the recovery of the ability of the spinal cord to produce stepping movements when facilitated by ES (Lavrov et al., 2006a).

To provide further insight into the role of afferents in generating stepping, we studied the recovery of stepping in complete spinal rats that also underwent an extensive unilateral deafferentation (T12-S2). We observed that ES-facilitated locomotion is mediated primarily by the afferents projecting to the ipsilateral locomotor networks, although modulation of sensory feedback from the non-deafferented side facilitated some long-duration 
cyclic movements on the deafferented side after a recovery period of 7 weeks.

Preliminary results have been published previously (Lavrov et al., 2006b).

\section{Materials and Methods}

Data were collected from two groups of adult female Sprague Dawley rats (270 -300 g body weight): control rats $(n=5)$, and rats with a complete midthoracic (approximately T8) spinal cord transection plus unilateral deafferentation (T12-S2) $(n=4)$. All experimental procedures complied with the guidelines of the National Institutes of Health Guide for the Care and Use of Laboratory Animals and were conducted in accordance with a protocol approved by the Animal Care Committee at the University of California, Los Angeles.

Surgical procedures. All surgeries were performed under aseptic conditions. The rats were anesthetized deeply by a combination of ketamine $(100 \mathrm{mg} / \mathrm{kg})$ and xylazine $(10 \mathrm{mg} / \mathrm{kg})$ administered intraperitoneally and were maintained at a surgical level with supplemental doses of ketamine as needed.

Headplug. A small incision was made at the midline of the skull. The muscles and fascia were retracted laterally, small grooves were made in the skull with a scalpel, and the skull was dried thoroughly. Two amphenol headplugs with Teflon-coated stainless steel wires (AS632; Cooner Wire) were securely attached to the skull with screws and dental cement as described previously (Roy et al., 1991; Ichiyama et al., 2005). A skin incision was made in the mid-dorsal region of the back (see below), and eight wires from the headplug were routed subcutaneously to the most distal end of the opening.

EMG implants. Skin and fascial incisions were made to expose the bellies of the medial gastrocnemius (MG) and tibialis anterior (TA) muscles bilaterally. Two wires were routed subcutaneously from the back incision to each muscle site. Bipolar intramuscular EMG electrodes were formed and secured into the midbelly of each muscle as described previously (Roy et al., 1991). The EMG wires were coiled near each implant site to provide stress relief. Stimulation through the headplug was used to verify the proper placement of the electrodes in each muscle.

Spinal cord transection, unilateral dorsal rhizotomy, and epidural stimulation electrode implants. A mid-dorsal skin incision was made between T6 and L4, and the paravertebral muscles were retracted as needed. A partial laminectomy was performed at the T8-T9 level, and the dura was opened longitudinally. Lidocaine (2-3 drops) was applied locally, and the spinal cord was completely transected at a midthoracic (approximately T8) level as described previously (Talmadge et al., 2002). A partial laminectomy was performed from T13 to L2, and the dura was opened from T12 to S1 and from S3 to S5. The dorsal roots on the left side were transected intradurally (as close to their exit from the spinal column as possible) from T12 to S2 (Fig. 1). Two wires from the headplug were passed subcutaneously to the most proximal end of the back incision. One Teflon-coated wire was passed to $S 1$, and a small notch was made in the Teflon coating $(\sim 0.5-1.0 \mathrm{~mm})$ on the surface toward the spinal cord and served as the stimulating electrode. The wire was affixed to the dura (Fig. 1, S1 spinal segment) at the midline using 9.0 sutures as described previously (Ichiyama et al., 2005). The appropriate position of the stimulating electrode was verified postmortem: connective tissue secured the electrode to the dura at the correct location on the spinal cord in all rats and thus ensured a stable position for all stimulation sessions. All wires (for EMG and ES) were coiled in the back region to provide stress relief. The Teflon coating was stripped from the distal centimeter of the second wire, which was then inserted subcutaneously in the back region and served as a common ground.

All incision areas were irrigated liberally with warm, sterile saline and closed in layers (i.e., investing fascia and then the skin). All closed incision sites were cleansed thoroughly with saline solution. Analgesia was provided by Buprenex $(0.5-1.0 \mathrm{mg} / \mathrm{kg}$, i.m.; three times per day). The analgesics were initiated before completion of the surgery and continued for a minimum of $2 \mathrm{~d}$. The rats were allowed to fully recover from anesthesia in an incubator. The rats were housed individually, and the bladders of the spinal rats were expressed manually three times per day for the

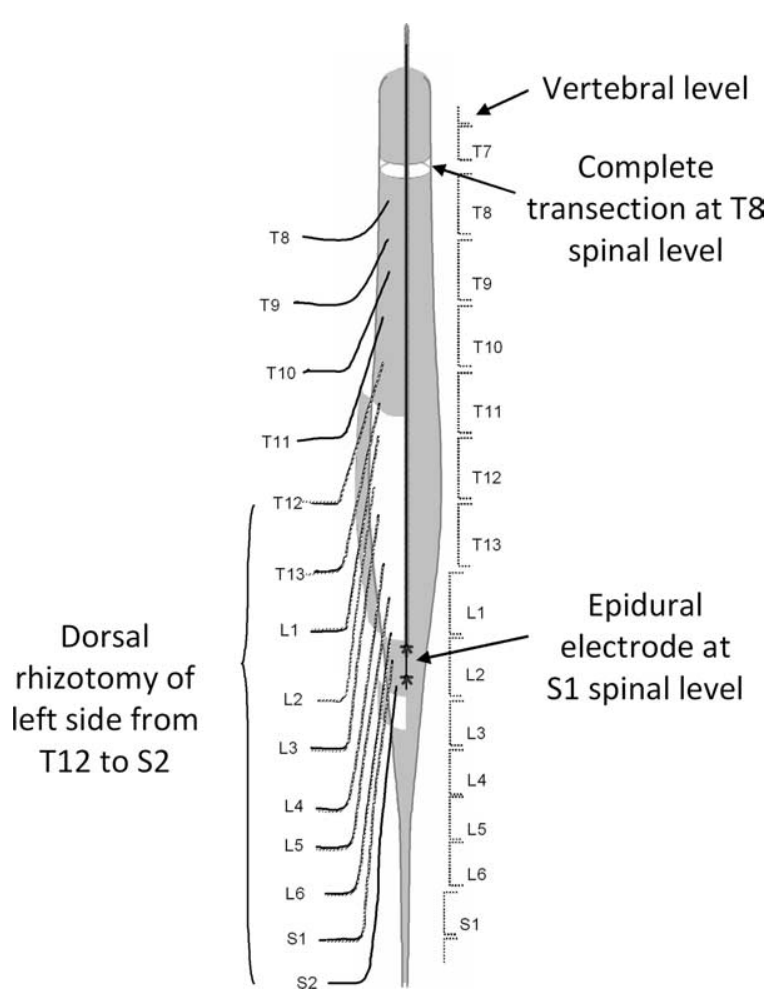

Figure 1. Schematic of the spinal cord transection and unilateral deafferentation surgery. $A$ complete spinal cord transection was performed at approximately the T8 spinal level (between vertebral segments $\mathrm{T7}$ and T8). The dura (gray shading) was opened longitudinally from vertebral level T11 to S3 (leaving a short strip at approximately the L2 level intact), and a unilateral (left side) dorsal rhizotomy (cutting the dorsal root as close to the spinal cord as possible and as close to its exit from the vertebral column as possible) was performed intradurally from the T12 to the $\$ 2$ spinal segments. A single insulated wire electrode with a small notch $(\sim 0.5-1.0 \mathrm{~mm})$ exposing the wire facing the spinal cord was sutured (asterisks represent the two sutures) on the short strip of intact dura over spinal segment $\$ 1$ through which electrical stimulation was used to facilitate stepping and induce bilateral reflex responses. The middle arrow points to the transection site, and the bottom arrow points to the epidural electrode implant site. The extent of the unilateral dorsal rhizotomy is identified on the left side with the cut dorsal roots shown as broken lines.

first 2 weeks after surgery and twice per day thereafter. The hindlimbs of the spinal rats were moved passively through a full range of motion once per day to maintain joint mobility. The motor responses to pinching of the toes were evaluated daily to test for the presence of afferent input. The procedures for the care of spinal animals have been detailed previously (Roy et al., 1992).

Stimulation and recording procedures. Testing procedures were started 1 week after surgery and were performed at 1,3 , and 7 weeks after surgery. All testing was performed weekly during ES with and without quipazine when the rats were fully awake. EMG activity was collected during stepping with a frequency of $2 \mathrm{kHz}$ using a custom-made Labview program, and the duration and mean amplitude of identified EMG bursts were determined (Roy et al., 1991; Courtine et al., 2008). The EMG signals were led from the animal through long shielded wires to amplifiers (A-M Systems) with a frequency response between $10 \mathrm{~Hz}$ and $1 \mathrm{kHz}$.

Stimulation was performed using a Grass S88 Stimulator and a stimulus isolation unit (Grass SIU5; both from Grass Instruments). Epidural stimulation at $\mathrm{S} 1$ was performed via single stimuli (duration, $0.5 \mathrm{~ms}$ ). Spinal cord reflexes were tested initially by stimulation at $0.2 \mathrm{~Hz}$ to determine the stimulus intensity-response amplitude curve. Averages of 10 responses were collected at stimulation intensities ranging from 0.5 to 10 $\mathrm{V}$ at $0.5 \mathrm{~V}$ intervals (Lavrov et al., 2006a).

To test for locomotor ability, the spinal rats were secured in an upper body harness support system, and the hindlimbs were placed on a moving treadmill belt as described previously (Ichiyama et al., 2005). Spinal 

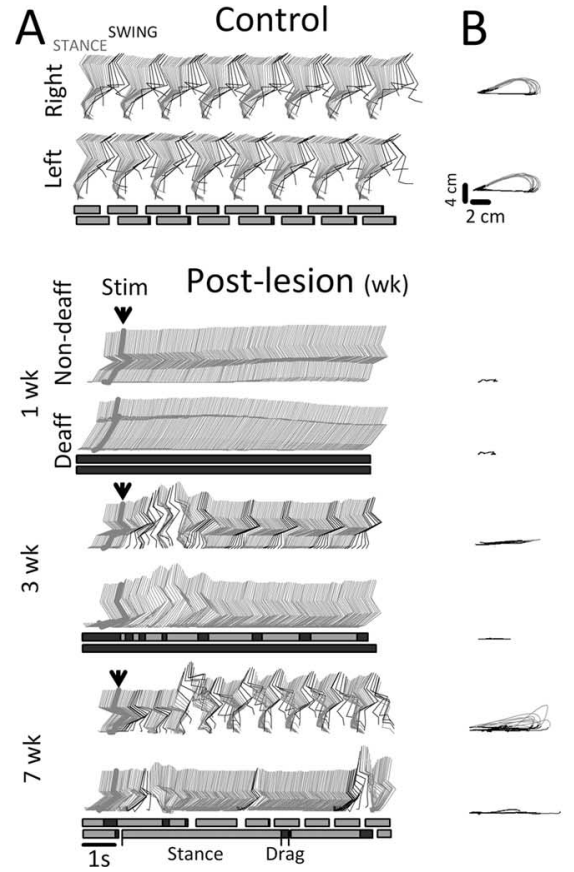
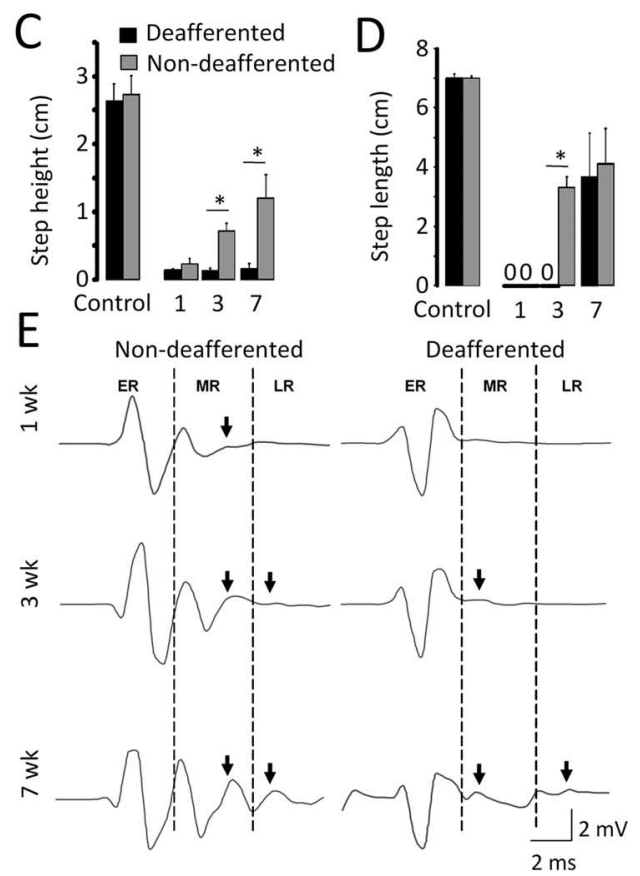

Figure 2. The rhythmicity of activity facilitated by epidural stimulation $(40 \mathrm{~Hz})$ and of spinal cord reflexes to a single stimulus after a complete midthoracic spinal cord transection and unilateral dorsal rhizotomy (Fig. 1). A, Representative stick diagrams of the hindlimb movements in a control rat and in an experimental rat, 1, 3, and 7 weeks after lesion. The time between individual sticks is $40 \mathrm{~ms}$. Blocks at the bottom of each pair of stick diagrams represent stance (gray), swing (blank), and foot dragging (black). Arrowheads indicate the initiation of epidural stimulation (Stim). Deaff, Deafferentation; Non-deaff, non-deafferentation. B, Successive trajectories of the right and left hindlimb endpoint recorded from the same rats and same steps as in $A$. C, D, Mean (SEM) step height $(\boldsymbol{C})$ and step length $(\boldsymbol{D})$ from both limbs of control and spinal-transected rats with unilateral deafferention. $\boldsymbol{E}$, Spinal cord motor-evoked responses to a single stimulus recorded from the tibialis anterior muscle on the non-deafferented and deafferented hindlimb of a representative spinal rat at 1,3, and 7 weeks after lesion. The stimulus (not shown) is at the beginning of each trace. The arrows indicate the appearance of $\mathrm{MR}$ or $\mathrm{LR} .{ }^{*} p<0.05$, significant difference between the non-deafferented and deafferented hindlimbs. All values for the non-deafferented and deafferented hindlimbs in $\boldsymbol{C}$ and $\boldsymbol{D}$ are statistically lower than control. ER, Early response. Error bars indicate SEM.

cord stimulation at $\mathrm{S} 1$ was induced by continuous ES at $40 \mathrm{~Hz}(25 \mathrm{~ms}$ interpulse interval) and a pulse duration of $0.2 \mathrm{~ms}$. At 7 weeks after surgery, to improve the stepping performance, the rats were injected with the 5 -HT agonist quipazine $(0.3 \mathrm{mg} / \mathrm{kg}$, i.p. $)$ as described previously (Ichiyama et al., 2005). An automated body weight support system was used to provide the optimum amount of body weight support necessary to enable walking (Ichiyama et al., 2005).

Analysis of reflex data. All recordings were performed and analyzed as described previously (Lavrov et al., 2006a). To identify the latencies and amplitudes of EMG responses to epidural stimulation, stimulus intensities between 0.5 and $10 \mathrm{~V}$ were used. During each test session, it was determined that three responses were modulated differentially and were clearly voltage dependent. We also used rate-dependent inhibition to separate the different spinal cord motor-evoked responses as described previously (Lavrov et al., 2006a). Short-latency (between 1.5 and $6.5 \mathrm{~ms}$ ) early responses remained unchanged under all frequencies of stimulation and correspond to direct activation of motoneurons. Middle (monosynaptic) responses with latencies between 6.5 and $9.5 \mathrm{~ms}$ were partially inhibited with 5 and $10 \mathrm{~Hz}$ stimulation. Late responses (LRs) with latencies between 9.5 and $13.5 \mathrm{~ms}$ that may reflect the activation of polysynaptic circuits were completely inhibited with 5 and $10 \mathrm{~Hz}$ stimulation (Gerasimenko et al., 2006; Lavrov et al., 2006a).

Kinematics recordings. Three-dimensional (3D) video recordings (100 $\mathrm{Hz}$ ) were made using four cameras (two cameras on each side at $100 \mathrm{fps}$; Basler Vision Technologies) oriented at 45 and $135^{\circ}$ with respect to the direction of the locomotion (i.e., the animal's sagittal plane on both sides). Reflective markers were attached bilaterally to the shaved skin overlying the following bony landmarks: the anterior superior iliac spine (Il), the greater trochanter (GT), the knee joint (K), the malleolus (M), the fifth metatarsal (MT), and the tip of toe (T). The SIMI motion capture software (SIMI Reality Motion Systems) was used to obtain 3D coordinates of the markers.

Stepping data analysis. The body was modeled as an interconnected chain of rigid segments (i.e., Il-GT for the pelvis, GT-K for the thigh, K-M for the shank, M-MT for the foot, and MT-T for the fifth digit), and the joint angles were generated as described previously (Courtine et al., 2005). A cycle was defined as the time interval between two successive paw contacts. Successive paw contacts were identified with an accuracy of \pm 1 video frame. Data for a duration to acquire $\sim 10$ step cycles on the non-deafferented side were extracted from a continuous sequence for each animal. Limb kinematics were determined as described by Courtine et al. (2005).

Muscle weights. At the end of the experimental period, the rats were deeply anesthetized with sodium pentobarbital $\left(125 \mathrm{mg} \cdot \mathrm{kg}^{-1}\right.$; Sigma) and transcardially perfused with $1 \mathrm{ml} \cdot \mathrm{g}^{-1}$ body weight of PBS, $\mathrm{pH} 7.4$, followed by $2 \mathrm{ml} \cdot \mathrm{g}^{-1}$ body weight of $4 \%$ paraformaldehyde in PBS (Sigma). The following muscles were removed bilaterally, cleaned of excess fat and connective tissue, and weighed: the soleus, MG, and plantaris as representative plantarflexors, and the TA and extensor digitorum longus as representative dorsiflexors.

Statistical analyses. All data are reported as mean \pm SEM. Statistically significant differences were determined using a one-way repeatedmeasures ANOVA. Values that were not normally distributed were analyzed using the nonparametric Kruskal-Wallis rank test. Paired Student's $t$ tests and nonparametric Wilcoxon tests were used to assess statistical differences between the left and right sides. The criterion level for statistical significance was set at $p<0.05$ for all analyses.

\section{Results}

Recovery of stepping ability after a complete spinal cord transection and unilateral rhizotomy

Recovery of stepping on the non-deafferented side

The ability of the spinal rats to step when provided with ES was tested 1, 3, and 7 weeks after injury. At 1 week, ES at $40 \mathrm{~Hz}$ applied at the $S 1$ spinal segment failed to produce any rhythmic activity in 
the hindlimb on the non-deafferented side (Fig. $2 A, B, 1$ week). Approximately 2 weeks after the injury, two to three consecutive step-like movements could be observed at the onset of ES (data not shown). Consistent sequences of stepping movements reappeared around week 3 only on the non-deafferented side (Fig. $2 A, B, 3$ weeks). These movements consisted of a stance phase on the dorsal aspect of the foot or with the toes curled, whereas the dorsum of the foot was mostly dragged along the treadmill belt during the swing phase. Stepping performance steadily improved during the following weeks, and at week 7 after injury, the animals showed stepping movements with plantar placement and less dragging of the paw during the swing phase of gait than at 3 weeks ( $52 \pm 5 \%$ of swing duration at 3 weeks vs $25 \pm 14 \%$ at 7 weeks) (Fig. $2 A, B$, compare 3 and 7 weeks). There was a progressive increase in both step height (Fig. $2 C$ ) and step length (Fig. $2 D$ ) over time, such that the mean step height was $43 \%$, and the mean step length was $49 \%$ of control values at the 7 week time point (supplemental Video 1, available at www.jneurosci.org as supplemental material). These values, however, were still lower than control values. In addition, step height was higher on the non-deafferented than on the deafferented side at 3 and 7 weeks after injury (Fig. 2C). At 3 weeks, step length was longer on the non-deafferented than on the deafferented side, but these values were similar at 7 weeks after injury (Fig. 2D).

\section{Recovery of movement on the deafferented side}

Despite the ES-enabled stepping activity on the non-deafferented side, no movement was observed on the deafferented side in any animal during the first $4-5$ weeks after injury. During this period, the deafferented hindlimb was hyperextended and dragged along the treadmill belt during stepping (Fig. 2A,B). Some steppinglike movements reappeared on the deafferented side $\sim 5-7$ weeks after the injury.

The movements of the deafferented hindlimb, however, occurred at a very low frequency in comparison with the stepping movements on the non-deafferented side. Based on limb kinematics, the mean cycle period with epidural stimulation alone was $6.72 \pm 2.13 \mathrm{~s}(n=3$; data from a total of only 7 steps, reflecting the very low frequency of occurrence relative to the intact side) on the deafferented side compared with $2.4 \pm 1.47 \mathrm{~s}$ ( $n=3$; data from 30 steps) on the non-deafferented side. These movements of the deafferented limb consisted of a long stance/ dragging period on the dorsum of the paw $(3.6 \pm 0.4 \mathrm{~s})$ in a hyperextended position followed by a very fast, kicking-like forward motion $(0.28 \pm 0.08 \mathrm{~s})$ that occurred most often after the beginning of the stance phase on the non-deafferented side (Fig. $2 A$; supplemental Video 1, available at www.jneurosci.org as supplemental material). Based on EMG patterns, the periodicity of the oscillations with a combination of epidural stimulation at S1 and quipazine was $8.85 \pm 1.15 \mathrm{~s}(n=3$; data from 28 steps $)$ on the deafferented side compared with $1 \pm 0.21 \mathrm{~s}(n=3$; data from 97 steps) on the non-deafferented side. All of these data illustrate the importance of ipsilateral afferent input in modulating stepping, whether it is based on kinematics or EMG and whether stepping is facilitated by ES alone or ES in combination with quipazine.

The EMG patterns on the deafferented side were characterized by long periods of very high tonic activity in the MG during stance/dragging followed by short, phasic EMG bursts in the TA during the fast forward-kicking movements (Fig. 3A). This pattern was in contrast to a more rhythmic and alternating bursting pattern on the non-deafferented side. The mean EMG amplitude was higher on the deafferented than on the non-deafferented side for both the MG and TA (Fig. 3B). In addition, the mean EMG

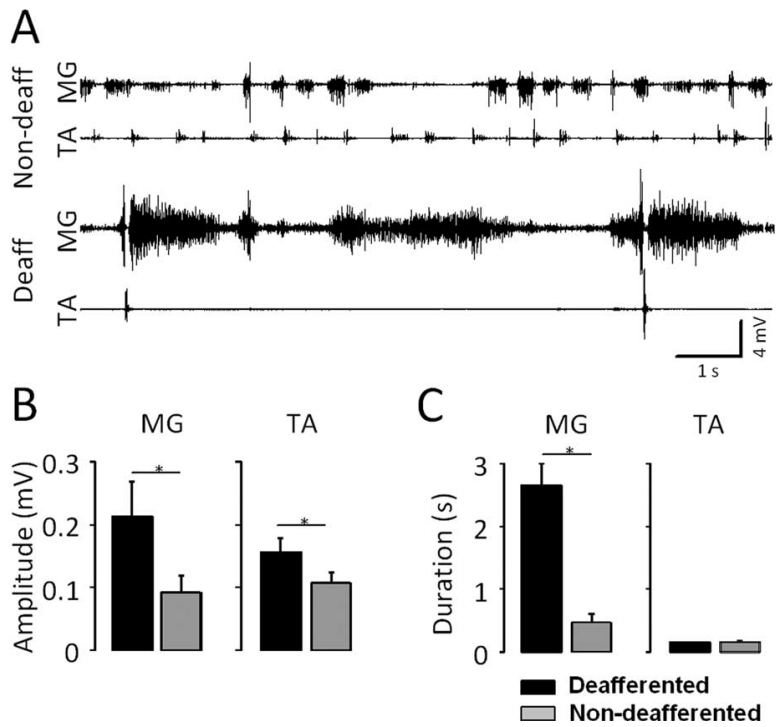

Figure 3. Analysis of EMG activity on the non-deafferented (Non-deaff) and deafferented (Deaff) sides at 7 weeks after a complete spinal cord transection and unilateral deafferentation. $A$, EMG bursting patterns for the MG and TA muscles on the non-deafferented and deafferented hindlimbs of a representative spinal rat during treadmill stepping at $7 \mathrm{~cm} / \mathrm{s}$ facilitated by epidural stimulation (at $\mathrm{S1}, 40 \mathrm{~Hz}$ ) and quipazine $(0.3 \mathrm{mg} / \mathrm{kg}$, i.p.) administration. $\boldsymbol{B}, \boldsymbol{C}$, Mean (SEM) EMG burst amplitude $(\boldsymbol{B})$ and duration $(\boldsymbol{C})$ for the MG and TA in the non-deafferented and deafferented hindlimbs for all rats. ${ }^{*} p<0.05$, significant difference between the nondeafferented and deafferented hindlimbs. Error bars indicate SEM.

burst duration was higher on the deafferented than on the nondeafferented side for the MG, but not different on the two sides for the TA (Fig. 3C).

Weight-bearing-related afferent input plays a critical role in the generation of step-like movements on the deafferented side

To investigate the role of afferent information during ES- and quipazine-enabled stepping, we studied the kinematics of both hindlimbs when the animals were positioned (suspended) above the treadmill belt without any weight-bearing afferent input (Fig. 4 , no contact) and when the hindlimbs were in contact with the treadmill belt and had weight-bearing afferent input (Fig. 4, contact). When the animal was suspended, a combination of quipazine and ES at S1 elicited step-like movements at a low frequency on the non-deafferented side, whereas the deafferented hindlimb remained hyperextended (supplemental Video 2, available at www.jneurosci.org as supplemental material). When the animal was lowered onto the moving treadmill belt, the contact of the hindlimb on the non-deafferented side stopped the ongoing airstepping movements, and rhythmic-stepping movements on the treadmill began within $2.3 \pm 0.85 \mathrm{~s}$ and continued until the termination of ES or treadmill belt motion. The onset of some slowly oscillating movements of the deafferented hindlimb at contact on the treadmill was delayed significantly $(7.1 \pm 2.4 \mathrm{~s})$, relative to the onset of locomotion on the non-deafferented side.

Restoration of flexion and cross-extension reflexes

Flexion and cross-extension reflexes were evaluated daily in both hindlimbs after surgery. There was no response to pinching of the paw on the deafferented side during the entire testing period. At 1 or $2 \mathrm{~d}$ after surgery, no animals responded to paw pinching on the non-deafferented side. At $3 \mathrm{~d}$ after surgery, pinching or pressing the paw on the non-deafferented side produced a weak ipsilateral flexor reflex but no response on the contralateral (deafferented) side. At 3-7 d after surgery, all animals showed a flexion 
response on the non-deafferented side and the appearance of a weak cross-extension reflex on the deafferented side.

\section{Restoration of spinal cord} motor-evoked responses

Early, middle, and late responses were evoked by single-shock stimulation of the spinal cord at S1 in all animals at 1, 3, and 7 weeks after surgery while the animals were in a body weight support system as described previously (Lavrov et al., 2006). An early response that reflects the direct activation of motor pools or motor axons was observed in both the MG and TA muscles on both sides at 1 week and did not change significantly during the remainder of the study. There was a gradual increase in the maximum amplitude of the middle response (MR) from 1 to 7 weeks after injury (Fig. $2 E$ ), with the $\mathrm{MR} / \mathrm{max}$ amplitude or the TA being significantly higher in the non-deafferented than in the deafferented hindlimb at 3 weeks (Fig. 5B). A late (long latency) response was observed on the non-deafferented side in both the MG and TA at 3 weeks and on the deafferented side at 7 weeks after surgery (except in the MG in one animal at 3 weeks) (Fig. 2E), with the LR/max amplitude being significantly higher in the non-deafferented than in the deafferented hindlimb at 3 and 7 weeks for the MG (Fig. 5C) and at 3 weeks for the TA (Fig. 5D).

\section{Muscle weights}

Considering that the extensive unilateral deafferentation (T12-S2) may be expected to result in muscle atrophy on the deafferented side and affect the responses evoked by spinal cord stimulation, the weights of several hindlimb muscles were determined bilaterally. There were no significant differences in the weights of the soleus, plantaris, TA, or extensor digitorum longus between the deafferented and nondeafferented hindlimbs. The weight of the MG was higher on the deafferented than on the non-deafferented side.

\section{Discussion}

The present data rather clearly demonstrate three important and novel points regarding the spinal control of locomotion. First, when facilitating bilateral stepping with epidural stimulation applied at the midline of the spinal cord, each leg is almost completely dependent on the presence of afferent projections on that same side. Despite the commonly recognized bilateral communication within the spinal circuitry even in fictive locomotion, the present results demonstrate a remarkable level of independence of each side when stepping is facilitated by epidural stimulation. Second, these results demonstrate a highly interdependent effect of electrical stimulation of the spinal afferents projecting to the spinal circuitry and the proprioceptive information that is derived nordeafferented hindlimbs.

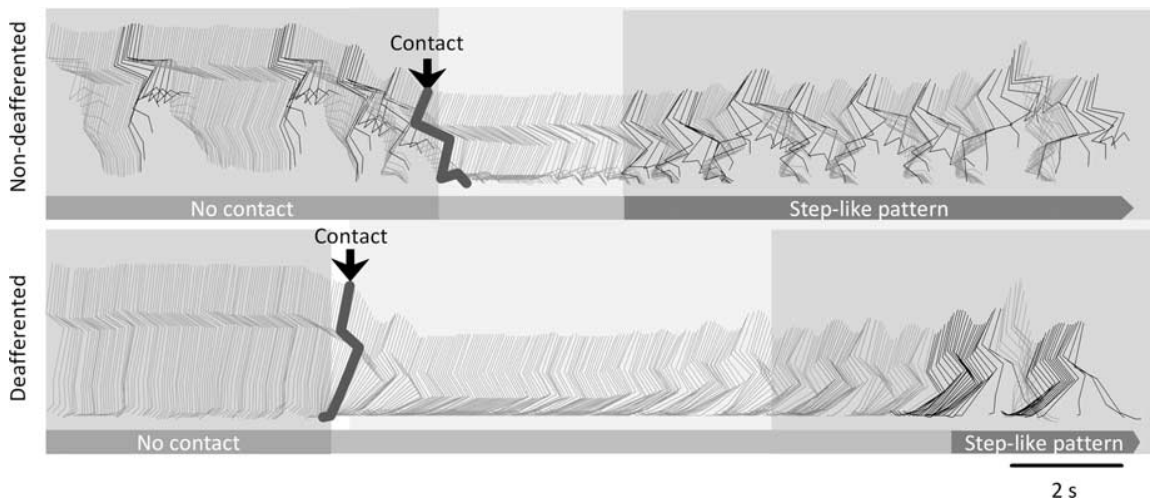

Figure 4. Intact afferents are required for the initiation of activity on the non-deafferented and deafferented sides in spinal rats. Representative stick diagrams are shown of the movements observed on the non-deaffererented and deafferented sides of a spinal rat while suspended above the treadmill (no foot contact) and after contact (arrows) of the feet with the moving treadmill belt when facilitated by epidural stimulation (at S1, $40 \mathrm{~Hz})$ and quipazine $(0.3 \mathrm{mg} / \mathrm{kg}$, i.p.) administration. The time between individual sticks is $40 \mathrm{~ms}$. Note the slow and delayed movements on the deafferented side occurring after rhythmic activity is initiated on the non-deafferented side.
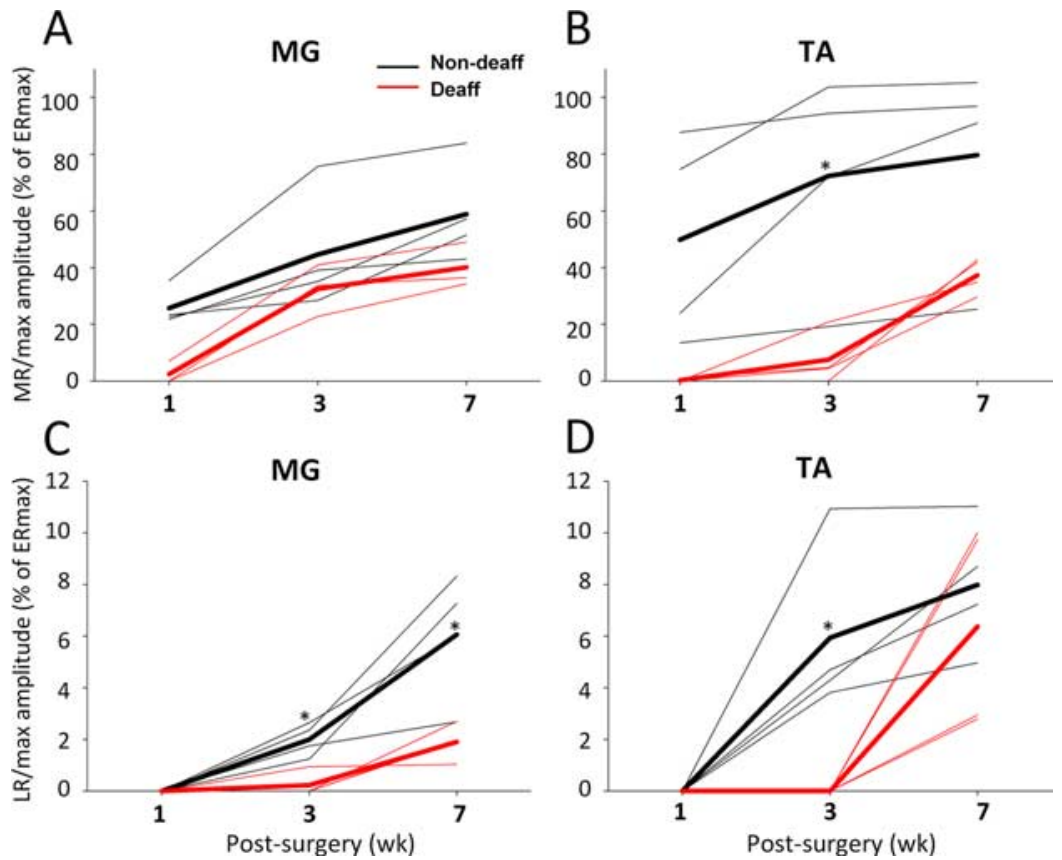

Figure 5. $\boldsymbol{A}-\boldsymbol{D}$, Changes in the maximum amplitude of the $M R(M R / \max )$ and $L R(L R / \max )$ for the $M G(\boldsymbol{A}, \boldsymbol{C})$ and $T A(\boldsymbol{B}, \boldsymbol{D})$ on the non-deafferented (black) and deafferented (red) sides of a spinal rat at 1,3, and 7 weeks after surgery. Data represent the average of 10 evoked potentials from four rats (thin lines) and the overall average for the four rats for each time point (thick lines). $\mathrm{MR} / \mathrm{max}$ and $\mathrm{LR} / \mathrm{max}$ are shown as a percentage of ERmax at each time point. Note that the amplitudes of the responses are consistently higher on the non-deafferented side and that a significant level of recovery of the $L R$ on the deafferented side occurs only at 7 weeks for both the MG and TA. ${ }^{*} p<0.05$, significant difference between the means for the non-deafferented and

mally from the hindlimbs when placed on a moving treadmill belt. Third, this complete dependence of the afferents on the same side to facilitate stepping can change over time via reorganization of the spinal circuitry that interprets the sensory input, such that afferents from the non-deafferented side can drive occasional movements on the deafferented side.

\section{Completeness of the unilateral deafferentation for the motor pools studied}

Both morphological and electrophysiological data locate the motor pools and sensory projections for the MG between the L4 and L6 spinal segments and for the TA between the L4 and L5 spinal 
segments (Vejsada and Hník, 1980; Peyronnard and Charron, 1983; Peyronnard et al., 1986; Manzano and McComas, 1988; Rivero-Melián, 1996). Axons of proprioceptive endings from the TA and extensor digitorum longus were found mainly at L4 with only a few at L5 and from the posterior superficial crural muscles (gastrocnemius, soleus, plantaris) mainly at L5 (Pantaleo and Calamai, 1973). Thus, unilateral deafferentation from the T12 to S2 spinal cord levels should eliminate sensory input to the motoneuronal pools tested. Our results demonstrate that a model of complete spinal cord transection and extensive unilateral deafferentation can be combined with chronically implanted epidural and intramuscular EMG electrodes for quantifying bilateral spinal cord synaptic efficacy in vivo in rats.

\section{ES facilitates stepping via spinal cord afferents}

In the present study, epidural stimulation at near motor thresholds facilitated stepping in all tested animals only in the nondeafferented hindlimb after the loss of the afferent input on one side. These results support our hypothesis that in spinal rats epidural stimulation facilitates spinal locomotor networks through the dorsal root afferents and are in agreement with previous modeling studies (Coburn, 1985; Coburn and Sin, 1985; Rattay, 1989; Holsheimer, 1998). The loss of sensory information from one side had little or no effect on the rhythmic activity on the non-deafferented side. Indeed, EMG and kinematics features from the non-deafferented side were remarkably similar to the stepping facilitated by epidural stimulation in spinal rats with intact afferents (Ichiyama et al., 2005; Lavrov et al., 2006a; Gerasimenko et al., 2007). These data emphasize the prominent role of ipsilateral projections in mediating the facilitation effect of epidural stimulation on stepping.

Recovery of the late (long latency) response evoked by epidural stimulation on the non-deafferented side was similar in amplitude and timing to the recovery of the late response previously observed in spinal rats with intact afferents (Lavrov et al., 2006a). Also, there appears to be some association with the timing of the restoration of locomotor-like movements facilitated by epidural stimulation and the reemergence of a late response. The time of recovery of reflex responses evoked by toe pinching $(\sim 7$ d) and spinal cord stimulation ( $\sim 2-3$ weeks) differed. The earlier restoration of ipsilaterally and contralaterally evoked responses from pinching of the intact limb may correspond to restoration of early components of flexor-extensor reflexes that also occurs within hours to days after spinal cord injury (Valero-Cabré et al., 2004). Recovery of the late response observed 3 weeks after spinal cord transection on the non-deafferented side corresponds to the restoration of polysynaptic reflexes mediated by $\mathrm{A} \alpha$ and $\mathrm{A} \beta$ lowthreshold skin afferents 2-3 weeks after injury (Valero-Cabré et al., 2004; Lavrov et al., 2006a). Although the effect of epidural stimulation at near motor thresholds appears to be primarily mediated by dorsal root afferents, the restoration of the middle response on the deafferented side after $\sim 3$ weeks suggests that stimulation also influences intraspinal fibers that have monosynaptic contact with motoneurons. These findings implicate additional synaptic events beyond the monosynaptic Ia afferents, i.e., the classical H-reflex, in the generation of the epidural stimulation-evoked middle response.

The transected spinal cord can adapt to the unilateral loss of afferent input via crossed connections from the non-deafferented side

The present results demonstrate that sensory information from the non-deafferented side can affect the spinal circuitry of the deafferented side (i.e., trigger spinal circuits on the deafferented side to generate an occasional robust movement). For all tested animals, the appearance of movement on the deafferented side was observed only after recovery of stepping on the nondeafferented hindlimb. Also, on the deafferented side, movement was only observed after paw contact of the non-deafferented hindlimb on a moving treadmill belt (i.e., only in the presence of robust stepping activity on the non-deafferented side). The activity observed on the deafferented side consisted of robust forward movements of the hindlimb. MG EMG burst amplitude and duration of the deafferented hindlimb was significantly greater than on the non-deafferented side. The prolonged duration of extensor (MG) EMG bursts during stance with no difference in the duration of the flexor (TA) EMG bursts during swing in the deafferented hindlimb bears some resemblance to the differential modulation of stance and swing when stepping at different speeds (Halbertsma 1983; Roy et al., 1991; Prochazka et al., 2002). Also, the short periods of inhibition of the extensor muscles on the deafferented side demonstrate a persistence of intralimb reciprocal inhibition within the spinal circuitry. These observations suggest that significant spinal reorganization of commissural connections occurred after unilateral dorsal rhizotomy. These results extend our previous findings that epidural stimulation alone does not necessarily induce stepping without placement of the paw on the treadmill (Ichiyama et al., 2005) and emphasizes that rather than inducing stepping, ES facilitates stepping when appropriate sensory input is provided (Edgerton, et al., 2004).

\section{References}

Coburn B (1985) A theoretical study of epidural electrical stimulation of the spinal cord-Part II: Effects on long myelinated fibers. IEEE Trans Biomed Eng 32:978-986.

Coburn B, Sin WK (1985) A theoretical study of epidural electrical stimulation of the spinal cord-Part I: Finite element analysis of stimulus fields. IEEE Trans Biomed Eng 32:971-977.

Courtine G, Roy RR, Hodgson J, McKay H, Raven J, Zhong H, Yang H, Tuszynski MH, Edgerton VR (2005) Kinematic and EMG determinants in quadrupedal locomotion of a non-human primate (Rhesus). J Neurophysiol 93:3127-3145.

Courtine G, Harkema SJ, Dy CJ, Gerasimenko YP, Dyhre-Poulsen P (2007) Modulation of multisegmental monosynaptic responses in a variety of leg muscles during walking and running in humans. J Physiol 582:1125-1139.

Courtine G, Song B, Roy RR, Zhong H, Herrmann JE, Ao Y, Qi J, Edgerton VR, Sofroniew MV (2008) Recovery of supraspinal control of stepping via indirect propriospinal relay connections after spinal cord injury. Nat Med 14:69-74.

Dimitrijevic MM, Dimitrijevic MR (2002) Clinical elements for the neuromuscular stimulation and functional electrical stimulation protocols in the practice of neurorehabilitation. Artif Organs 26:256-259.

Dimitrijevic MR, Gerasimenko Y, Pinter MM (1998) Evidence for a spinal central pattern generator in humans. Ann N Y Acad Sci 860:360-376.

Edgerton VR, Roy RR, Hodgson JA, Gregor RJ, de Guzman CP (1991) Recovery of full weight-supporting locomotion of the hindlimbs after complete thoracic spinalization of adult and neonatal cats. In: Restorative Neurology, Vol 5, Plasticity of motoneuronal connections (Wernig A, ed), pp 405-418. New York: Elsevier.

Edgerton VR, Leon RD, Harkema SJ, Hodgson JA, London N, Reinkensmeyer DJ, Roy RR, Talmadge RJ, Tillakaratne NJ, Timoszyk W, Tobin A (2001) Retraining the injured spinal cord. J Physiol 533:15-22.

Edgerton VR, Tillakaratne NJ, Bigbee AJ, de Leon RD, Roy RR (2004) Locomotor recovery potential after spinal cord injury. In: Neurobehavioral determinants of interlimb coordination (Swinnen SP, Duysens J, eds), pp 53-91. Dordrecht, The Netherlands: Kluwer.

Gerasimenko YP, Avelev VD, Nikitin OA, Lavrov IA (2003) Initiation of locomotor activity in spinal cats by epidural stimulation of the spinal cord. Neurosci Behav Physiol 33:247-254.

Gerasimenko YP, Lavrov IA, Courtine G, Ichiyama RM, Dy CJ, Zhong H, Roy RR, Edgerton VR (2006) Spinal cord reflexes induced by epidural spinal cord stimulation in normal awake rats. J Neurosci Methods 157:253-263. 
Gerasimenko YP, Ichiyama RM, Lavrov IA, Courtine G, Cai L, Zhong H, Roy RR, Edgerton VR (2007) Epidural spinal cord stimulation plus quipazine administration enable stepping in complete spinal adult rats. J Neurophysiol 98:2525-2536.

Goldberger ME (1988) Partial and complete deafferentation of cat hindlimb: the contribution of behavioral substitution to recovery of motor function. Exp Brain Res 73:343-353.

Halbertsma JM (1983) The stride cycle of the cat: the modelling of locomotion by computerized analysis of automatic recordings. Acta Physiol Scand Suppl 521:1-75.

Herman R, He J, D’Luzansky S, Willis W, Dilli S (2002) Spinal cord stimulation facilitates functional walking in a chronic, incomplete spinal cord injured. Spinal Cord 40:65-68.

Holsheimer J (1998) Computer modelling of spinal cord stimulation and its contribution to therapeutic efficacy. Spinal Cord 36:531-540.

Ichiyama RM, Gerasimenko YP, Zhong H, Roy RR, Edgerton VR (2005) Hindlimb stepping movements in complete spinal rats induced by epidural spinal cord stimulation. Neurosci Lett 383:339-344.

Lavrov I, Gerasimenko YP, Ichiyama RM, Courtine G, Zhong H, Roy RR, Edgerton VR (2006a) Plasticity of spinal cord reflexes after a complete transection in adult rats: relationship to stepping ability. J Neurophysiol 96:1699-1710.

Lavrov I, Dy CJ, Van den Brand R, Zhong H, Roy RR, Edgerton VR (2006b) Spinal cord reorganization in chronic spinal rats with unilateral deafferentation. Soc Neurosci Abstr 32:447.9.

Manzano G, McComas AJ (1988) Longitudinal structure and innervation of two mammalian hindlimb muscles. Muscle Nerve 11:1115-1122.

Pantaleo T, Calamai F (1973) Afferent proprioreceptive fibers in the rat and their distribution in the dorsal roots. Arch Fisiol 70:133-148.

Peyronnard JM, Charron L (1983) Motoneuronal and motor axonal inner- vation in the rat hindlimb: a comparative study using horseradish peroxidase. Exp Brain Res 50:125-132.

Peyronnard JM, Charron LF, Lavoie J, Messier JP (1986) Motor, sympathetic and sensory innervation of rat skeletal muscles. Brain Res 373:288-302.

Prochazka A, Gritsenko V, Yakovenko S (2002) Sensory control of locomotion: reflexes versus higher-level control. Adv Exp Med Biol 508:357-367.

Rattay F (1989) Analysis of models for extracellular fiber stimulation. IEEE Trans Biomed Eng 36:676-682.

Rivero-Melián C (1996) Organization of hindlimb nerve projections to the rat spinal cord: a choleragenoid horseradish peroxidase study. J Comp Neurol 364:651-663.

Rossignol S (2006) Plasticity of connections underlying locomotor recovery after central and/or peripheral lesions in the adult mammals. Philos Trans R Soc Lond B Biol Sci 361:1647-1671.

Roy RR, Hutchison DL, Pierotti DJ, Hodgson JA, Edgerton VR (1991) EMG patterns of rat ankle extensors and flexors during treadmill locomotion and swimming. J Appl Physiol 70:2522-2529.

Roy RR, Hodgson JA, Lauretz SD, Pierotti DJ, Gayek RJ, Edgerton VR (1992) Chronic spinal cord-injured cats: surgical procedures and management. Lab Anim Sci 42:335-343.

Talmadge RJ, Roy RR, Caiozzo VJ, Edgerton VR (2002) Mechanical properties of rat soleus after long-term spinal cord transection. J Appl Physiol 93:1487-1497.

Valero-Cabré A, Forés J, Navarro X (2004) Reorganization of reflex responses mediated by different afferent sensory fibers after spinal cord transection. J Neurophysiol 91:2838-2848.

Vejsada R, Hník P (1980) Radicular innervation of hindlimb muscles of the rat. Physiol Bohemoslov 29:385-392. 